\title{
Erratum: "Representative strain of indentation analysis" [J. Mater. Res. 20, 2225 (2005)] and "Limit analysis-based approach to determine the material plastic properties with conical indentation" [J. Mater. Res. 21, 947 (2006)]
}

\author{
Nagahisa Ogasawara and Norimasa Chiba \\ Department of Mechanical Engineering, National Defense Academy, Hashirimizu, \\ Yokosuka 239, Japan \\ $\mathrm{Xi} \mathrm{Chen}{ }^{\mathrm{a}}$ \\ Department of Civil Engineering and Engineering Mechanics, Columbia University, \\ New York, New York 10027
}

(Received 14 July 2006; accepted 19 July 2006)

We like to take this opportunity to rectify an error in the schematic drawing in two of our recently published research articles. ${ }^{1,2}$ A typographical error of a number appeared in Fig. 5 of J. Mater. Res. 20, 2225 (2005), p. $2229^{1}$ and in Fig. 2 of J. Mater. Res. 21, 947 (2006), p. 948²: these two figures are the same schematic drawing. In both figures, the number " 1 " on the slopes should be changed to "2." The correct figure is given as Fig. 1 in this erratum.

Incidentally, this error may have contributed to a misunderstanding of our article in the recent analysis by Cao and Huber [J. Mater. Res., 21, 1810 (2006)], ${ }^{3}$ who used the factor "1" instead of "2" based on the incorrect figure to calculate the representative stress. A comment ${ }^{4}$ of this paper is forthcoming (in which we also comment on the new technique developed by Cao and $\mathrm{Huber}^{3}$ ). In part of another paper, ${ }^{5}$ we also showed in detail how we derived the factor "2" (such information was not included in Ref. 1).

We would like to take this opportunity to clarify the correct formula we used to compute the representative strain and stress in both articles. ${ }^{1,2}$ For Berkovich indenter, the representative strain and the representative stress are calculated as

$$
\epsilon_{R}=0.0115 \text { and } \sigma_{R}\left\langle\epsilon_{R}\right\rangle=R\left(2 \cdot \epsilon_{R}+2 \cdot \sigma_{R}\left\langle\epsilon_{R}\right\rangle / E\right)^{n}
$$

respectively. Here, $E$ is the Young's modulus of the specimen and $n$ is the work-hardening exponent for a power-law hardening metal or alloy. When the indenter angle is changed in a moderate range, the value of $\epsilon_{R}$ varies as $\epsilon_{R}=$ $0.0319 \cdot \cot \alpha$, where $\alpha$ is the half-apex angle of the conical indenter, which is shown as Eq. (23) in Ref. 1, and the representative stress is still computed through Eq. (1) in this erratum. In our articles, the representative strain was assigned a physical meaning as the plastic strain in the biaxial stress-strain curve (Fig. 1 in this Comment) by assuming that the Poisson's effect is small. Note that neither this interpretation nor the incorrect figures have any effect on the numerical results presented in our articles. ${ }^{1,2}$ We have recently verified the accuracy of all numerical results in Refs. 1 and 2 , which will be discussed and compared with Cao and Huber's new technique ${ }^{3}$ in the Comment. ${ }^{4}$

In addition, we also note that Eq. (5) in Ref. 2 should be $\sigma_{R}\left\langle\epsilon_{R}\right\rangle=R\left(2 \epsilon_{a}^{e}+2 \epsilon_{R}\right)^{n}$ where the equal sign was missing. Eq. (30) in Ref. 2 should be $\sigma=\sigma_{R}\left\langle\epsilon_{R}\right\rangle$ and $\epsilon=2 \cdot\left(\epsilon_{R}+\frac{\sigma_{R}\left\langle\epsilon_{R}\right\rangle}{E}\right)$ where the parenthesis was missing. These missing symbols were in our original manuscript but got lost in the final version.

\footnotetext{
${ }^{a)}$ Address all correspondence to this author.

e-mail: xichen@civil.columbia.edu

DOI: 10.1557/JMR.2006.0319
} 


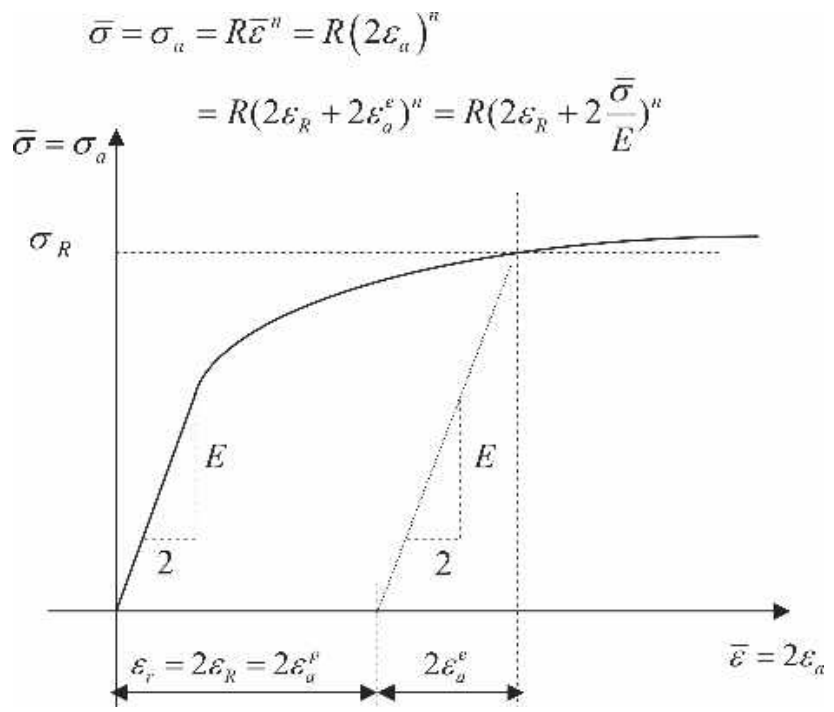

FIG. 1. Definition of the representative strain $\epsilon_{R}$ and the representative stress $\sigma_{R}\left\langle\epsilon_{R}\right\rangle$ on the biaxial stress-strain curve, which is used in Refs. 1 and 2 .

\section{REFERENCES}

1. N. Ogasawara, N. Chiba, and X. Chen: Representative strain of indentation analysis. J. Mater. Res. 20, 2225 (2005).

2. N. Ogasawara, N. Chiba, and X. Chen: Limit analysis-based approach to determine the material plastic properties with conical indentation. J. Mater. Res. 21, 947 (2006).

3. Y. Cao and N. Huber: Further investigation on the definition of the representative strain in conical indentation. J. Mater. Res. 21, 1810 (2006).

4. N. Ogasawara, N. Chiba, M. Zhao, and X. Chen: Comments on "Further investigation on the definition of the representative strain in conical indentation" by Y. Cao and N. Huber [J. Mater. Res. 21, 1810, (2006)], J. Mater. Res. (2006) (submitted).

5. N. Ogasawara, N. Chiba, M. Zhao, and X. Chen: Determine material plastic and elastic properties with optimized representative strain-based indentation technique. J. Mech. Mater. Struct. (2006) (submitted). 